\title{
Arcabouço de Formação de Pares para Online Peer Assessment
}

\author{
Leonam C. Oliveira1 ${ }^{1}$, Wellton C. Oliveira ${ }^{1}$, Selma S. Rosa ${ }^{2}$, Andrey R. Pimentel \\ ${ }^{1}$ Departamento de Informática - Universidade Federal do Paraná (UFPR) \\ Curitiba - PR - Brasil \\ ${ }^{2}$ Campus Avançado de Jandaia do Sul - Universidade Federal do Paraná (UFPR) \\ Jandaia do Sul - PR - Brasil \\ leonamcdeoliveira@gmail.com, \{wcoliveira, andrey\}@inf.ufpr.br, \\ selmasantoseufpr.br
}

\begin{abstract}
Online Peer Assessment (OPA) is a subject in recent studies about its validity in the teaching and learning process. Controversies are found in the literature, with students reporting discomfort during assessments. This research proposed the development of a framework to formation of heterogeneous pairs for OPA based on characteristics and attributes. A case study was conducted to understand the impact of this type of peer formation for better student acceptance during assessments. Greater comfort was detected during the assessment by students and also an indication of the contribution of the OPA to the student focused on the production, not feedback, of the evaluation.
\end{abstract}

Resumo. Online Peer Assessment (OPA) é alvo de estudos recentes quanto a sua validade dentro do processo de ensino e de aprendizagem. Controvérsias são encontradas na literatura, com o relato de estudantes quanto a sentirem desconforto durante as avaliações. Esta pesquisa propôs o desenvolvimento de um arcabouço para a formação de pares heterogêneos para a OPA com base em características e atributos. Um experimento foi realizado para compreender o impacto deste tipo de formação de pares para uma melhor aceitação dos estudantes durante as avaliações. Foi detectado maior conforto durante a avaliação pelos estudantes e também um indício da contribuição da OPA para o estudante focada na produção, e não no feedback, da avaliação.

\section{Introdução}

Há algum tempo, a educação investiga a eficácia da aprendizagem colaborativa com o objetivo de melhorar o aprendizado dos estudantes [Gokhale, 2012], situação em que dois ou mais estudantes buscam o aprendizado em conjunto [Dillenbourg, 1999]. A aprendizagem colaborativa é voltada para o desenvolvimento do pensamento crítico para a resolução de problemas, sendo amplamente pesquisada e defendida por pesquisadores [Gokhale, 2012]. A aprendizagem colaborativa com suporte educacional, em inglês Computer-Supported Collaborative Learning (CSCL), propõe o desenvolvimento de softwares, que funcionem como mediadores dos estudantes, propiciando a realização da aprendizagem colaborativa [Stahl et al., 2006]. 
VIII Congresso Brasileiro de Informática na Educação (CBIE 2019)

Anais do XXX Simpósio Brasileiro de Informática na Educação (SBIE 2019)

Na busca por mudanças para melhorar o processo de ensino e de aprendizagem, há a necessidade de reestruturação e revisão dos fundamentos e práticas quanto à avaliação da aprendizagem dos educandos [Garcia, 2009]. Considera-se que a avaliação tem grande importância no contexto educacional, assim como há a necessidade do desenvolvimento de novas estratégias de avaliação [Garcia, 2009]. Tendo a necessidade de que a aprendizagem seja mais centrada no estudante [Pereira et al., 2010], uma avaliação alternativa é considerada uma avaliação mais humanizada, mais participativa e com integração entre os processos de ensino e de aprendizagem [Fernandes, 2005].

Online Peer Assessment (OPA), em português avaliação online por pares, é uma forma alternativa de avaliação que objetiva o aprendizado a partir da realização da avaliação dos colegas pelo próprio estudante [Miao e Koper, 2007], podendo ter papel tanto de par avaliado (quando recebe avaliações) quanto de par avaliador (quando realiza avaliações). Entre os benefícios da OPA, há o aumento do desempenho e do esforço do estudante, tornando a avaliação um incentivo para melhorar o trabalho em grupo [Sáiz et al., 2012]. Além disto, considera-se que há melhora na motivação, tornando os estudantes protagonistas de suas próprias avaliações [Sáiz et al., 2012].

Porém há controvérsias no processo e, dentre as limitações, pesquisas sugerem que há uma certa ansiedade dos estudantes com a OPA [Topping, 2009]. Há, portanto, dificuldade de estudantes acreditarem na justiça do processo [To, 2016] e também não se sentirem confiantes por considerarem que não estão preparados para realizar a avaliação [Cassidy, 2007]. Há relatos de estudantes que afirmam sentir desconforto ao avaliar os trabalhos de seus pares [Chen et al., 2010] e também ao terem seus trabalhos avaliados por outros estudantes [Wen e Tsai, 2008]. Busca-se reduzir o desconforto de estudantes e melhorar sua participação nos processos da OPA.

Entre as etapas da OPA, a formação dos pares recebe pouca atenção da literatura da área. Parte dos estudos não especifica a estratégia utilizada para a formação por pares [Sitthiworachart e Joy, 2008; Van der Pol et al., 2008; Ma, 2009; Yang e Tsai, 2010; Kaufman e Schunn, 2011; Li et al., 2012; Lai, 2016]. Esta pesquisa buscou propor o desenvolvimento de um arcabouço para realizar a formação de pares de estudantes, chamado de AFP-OPA(Arcabouço de Formação de Pares-OPA), baseando-se em atributos e características dos estudantes.

\section{Trabalhos relacionados}

Dentre os estudos da OPA mapeados, apenas um estudo utilizava atributos, neste caso as médias dos estudantes, para a formação dos pares [Demir, 2018]. Os trabalhos relacionados para a definição dos atributos e características, assim como as técnicas e algoritmos para a aplicação no AFP-OPA, partiram da formação de grupos no contexto da aprendizagem colaborativa com suporte computacional. As características e/ou atributos selecionados para o agrupamento foram perfil de personalidade [Balmaceda et al., 2014; Reis et al., 2018] e notas [Ho et al., 2009; Khandaker e Soh, 2010; Webber e Lima, 2012; Kardan e Sadeghi, 2016; Maina et al., 2017; Acharya e Sinha, 2018].

As escolhas das características e atributos foram definidas com base nos dados que poderiam ser identificados a partir da utilização dos estudantes no software "OPA!", que são as notas nas atividades, além de uma informação adicional, perfil de personalidade, que tem se mostrado relevante em estudos recentes para a formação de 
VIII Congresso Brasileiro de Informática na Educação (CBIE 2019)

Anais do XXX Simpósio Brasileiro de Informática na Educação (SBIE 2019)

grupos [Reis et al., 2018]. Para reunir as informações das características e atributos, dentre as tecnologias computacionais, foi possível detectar que algoritmos de agrupamento, neste caso o $K$-Means, que é um dos algoritmos comumente utilizado nas pesquisas [Webber e Lima, 2012; Maina et al., 2017; Acharya e Sinha, 2018], funcionando para agrupar estudantes em similaridade de notas.

O AFP-OPA prevê a divisão dos estudantes em grupos com notas similares, a partir do k-means, utilizado para particionar um conjunto de dados em $\mathrm{k}$ grupos [Wagstaff et al., 2001] e aplicação de questionário de perfil de personalidade a partir do modelo MBTI (Myers-Briggs Type Indicator), que é um indicador dos tipos psicológicos, auxiliando na identificação de preferências pessoais mais significativas [Couto et al., 2016]. Na seção seguinte, a arquitetura do AFP-OPA é apresentada com a integração entre as técnicas para a organização dos pares heterogeneamente.

\section{Arcabouço de formação de pares}

O AFP-OPA está inserido dentro de um software, chamado de "OPA!", que foi desenvolvido para a realização da OPA. Destaca-se que a avaliação no "OPA!" é anônima e ocorre a partir de rubricas, que são critérios pré-estabelecidos pelo docente para medir a qualidade de produtos dentro de uma escala (ruim a ótimo, por exemplo). Há também caixas para comentários, sendo esta de uso opcional.

Seguindo as mesmas especificações do software "OPA!", o AFP-OPA foi desenvolvido na linguagem PHP e banco de dados na linguagem SQL. O AFP-OPA atua como uma terceira estratégia de associação dos pares no "OPA!", além da associação aleatória e manual, já prévias do software. Conforme a Figura 3, a arquitetura foi proposta a partir das informações coletadas no k-means, que agrupa as notas dos estudantes em 4 grupos, definido com base em [Webber e Lima, 2012], e pelo questionário do modelo MBTI, que detecta o perfil de personalidade do estudante.

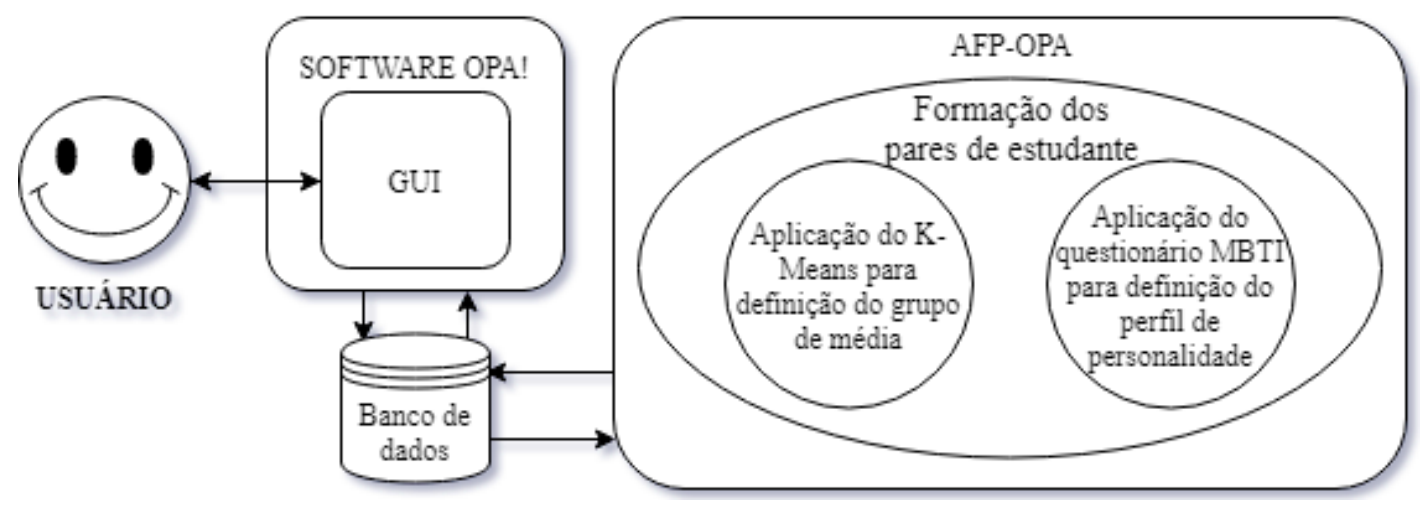

Figura 1. Arquitetura do AFP-OPA

Cada estudante é associado a 4 estudantes que serão seus avaliadores. A partir das informações de notas e perfis de personalidades de cada estudante, o AFP-OPA faz uma busca objetivando associar, para cada estudante, 4 pares avaliadores que tenham características diferentes entre si. Buscou-se formar os pares avaliadores heterogeneamente, considerando os estudos que afirmam as vantagens do agrupamento heterogêneo dos estudantes [Smith e Spindle, 2007]. Foi definida a quantidade de 4 pares avaliadores para cada estudante a partir de estudos que afirmam que a OPA tornase relevante caso um estudante receba ao menos 4 avaliações [Bouzidi e Jaillet, 2009] 
VIII Congresso Brasileiro de Informática na Educação (CBIE 2019)

Anais do XXX Simpósio Brasileiro de Informática na Educação (SBIE 2019)

As médias de estudantes e seus perfis de personalidade são diversos e podem não seguir um padrão, variando a quantidade de estudantes em cada grupo de média do $k$-means e também seus perfis de personalidade. Caso não seja possível associar para um estudante 4 pares avaliadores que sejam heterogêneos quanto ao grupo de média e o perfil de personalidade, novas buscas são realizadas. Utiliza-se então o cálculo da distância euclidiana para associar pares que estejam o mais distante possível dos outros pares avaliadores, mesmo que estejam no mesmo grupo de média do k-means.

\section{Metodologia}

Com o experimento buscou-se aplicar a pesquisa em um contexto de sala de aula do ensino superior, objetivando responder duas perguntas de pesquisa (PP1 e PP2) de pesquisa definidas a partir da fundamentação teórica deste estudo. A metodologia segue 5 passos, conforme definidos na Figura 1, que são exemplificados a seguir.

PP1: Formar pares heterogêneos a partir de atributos e características específicas pode melhorar a confiança dos estudantes, diminuindo o desconforto no processo da Online Peer Assessment.

PP2: Considerar atributos e características do estudante na formação de pares avaliadores para a OPA contribui para o desempenho acadêmico, sob a perspectiva discente, quanto à atividade avaliada.

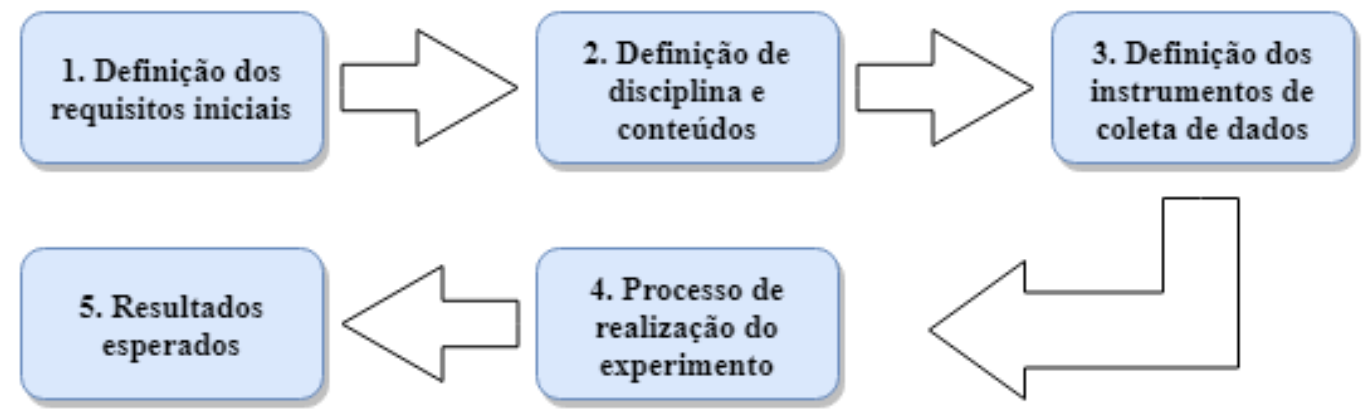

Figura 2. Metodologia do Experimento

O $1^{\circ}$ passo buscou a definição das necessidades para a aplicação do experimento. O universo de aplicação foi sala de aula, de forma presencial, em curso de ensino superior, para que realizassem uma atividade dentro do processo da OPA. A partir da disponibilidade, foram selecionadas duas turmas com igual conteúdo programático. Sendo as turmas de turnos diferentes e o experimento ocorrendo durante a aula, foi necessário definir uma turma como controle (sem o AFP-OPA) e uma turma como experimental (com o AFP-OPA), para realizar a comparação de resultados.

O $2^{\circ}$ passo definiu a disciplina com base nos requisitos iniciais elencados. Duas turmas da disciplina de Introdução à Programação, do curso de Manutenção Industrial de uma instituição pública de ensino, do mesmo docente, foram convidadas a participar do estudo. Foi solicitado aos estudantes, como atividade, que realizassem uma descrição narrativa de um problema proposto, sendo definida em conversa com o docente.

Para a coleta de dados ( $3^{\circ}$ passo), seguiu-se as orientações de [Coutinho, 2011]. Recorreu-se à técnicas e instrumentos diversificados: (a) questionário, contendo 8 
VIII Congresso Brasileiro de Informática na Educação (CBIE 2019)

Anais do XXX Simpósio Brasileiro de Informática na Educação (SBIE 2019)

questões (6 com escala likert e 2 questões abertas) e (b) análise documental (dados gerados em uma avaliação dos estudantes).

O $4^{\circ}$ passo definiu o processo para a aplicação do experimento, tendo sido proposto nesta ordem: $1^{\circ}$ ) conversa inicial com os estudantes; $2^{\circ}$ ) registro no software "OPA!"; $3^{\circ}$ ) responder ao questionário MBTI (perfil de personalidade); $4^{\circ}$ ) realização da atividade; $5^{\circ}$ ) avaliação dos pares; $6^{\circ}$ ) visualização do feedback; e $7^{\circ}$ ) os estudantes responderam ao questionário de avaliação quanto a sua percepção sobre o processo.

Entre os resultados esperados ( $5^{\circ}$ passo), acreditava-se que os estudantes do grupo experimental sentiriam maior conforto ao avaliar e serem avaliados pelos pares e que o feedback recebido seria considerado por eles mais relevante e mais variado dentre as avaliações que recebem no grupo experimental.

\section{Resultados e discussões}

Participaram do experimento 32 estudantes no grupo de controle e 31 estudantes no grupo experimental. Realizado em laboratórios de informática, os estudantes tinham acesso a um computador individual para realizarem a atividade. A variação entre o grupo experimental e o de controle foi a estratégia utilizada na formação dos pares. No grupo experimental os pares foram associados a partir da proposta do AFP-OPA (utilizando as notas na primeira prova da disciplina como média). No grupo de controle os pares foram formados aleatoriamente. Cada estudante, como par avaliado, foi associado a 4 pares avaliadores, e cada estudante avaliava a tarefa de 4 colegas.

Destaca-se que, apesar da estratégia de formação aleatória não garantir a homogeneidade (que seria o oposto do que o AFP-OPA busca formar), os pares formados apresentam 46,8\% de casos onde pelo menos dois dos quatro pares avaliadores de cada par avaliado possuía mesmo perfil de personalidade. Em compensação, no grupo experimental, todos os pares avaliadores de cada par avaliado possuíam perfis de personalidade distintos. Com relação aos resultados obtidos, a pergunta inicial questionava os estudantes se consideravam que o software "OPA!" auxiliava na realização da avaliação. Em ambos os grupos, os estudantes concordaram em sua maioria, sendo $90 \%$ no grupo de controle e $97 \%$ no grupo experimental.

Conforme a primeira pergunta de pesquisa formulada, a Tabela 1 traz os resultados das questões feitas aos estudantes para compreender a percepção discente quanto ao conforto no processo da OPA. Observa-se que os estudantes concordaram que se sentiram mais confortáveis com as pessoas escolhidas para que lhes avaliassem e as que eles avaliaram no grupo experimental. $75 \%$ concordou que se sentiram confortáveis com a maneira que ocorreu a formação de pares no grupo experimental, frente a $69 \%$ no grupo controle. Além disso o grupo controle obteve mais pessoas que discordavam da organização dos pares (12\%) contra o grupo experimental (9\%).

Com relação ao conforto no papel de par avaliador, os estudantes sentiram-se confortáveis em avaliar seus pares em $81 \%$ dos casos no grupo experimental e $72 \%$ dos casos no grupo controle. Novamente em relação a discordar do conforto ao avaliar os pares, o grupo controle apresentou $6 \%$ enquanto o grupo experimental $3 \%$. No papel de par avaliado os grupos (controle e experimental) tiveram resultados semelhantes quanto a concordar que se sentiram confortáveis em serem avaliados $(69 \%$ e $68 \%$, 
VIII Congresso Brasileiro de Informática na Educação (CBIE 2019)

Anais do XXX Simpósio Brasileiro de Informática na Educação (SBIE 2019)

respectivamente). Porém o grupo de controle discordou em maior número quanto ao conforto de ser avaliado, sendo $12 \%$ frente a $6 \%$ do grupo experimental. Conforme a PP1, é possível verificar um indício de conforto maior no grupo experimental.

Tabela 1. Resultados da Pergunta de Pesquisa 1

\begin{tabular}{|c|c|c|c|c|c|}
\hline \multicolumn{6}{|c|}{$\begin{array}{c}\text { Questão } 2 \text { - Você se sentiu confortável com a maneira que a organização dos pares ocorreu (as } \\
\text { pessoas que você avaliou e as pessoas que lhe avaliaram)? }\end{array}$} \\
\hline & $\begin{array}{l}\text { Concordo } \\
\text { totalmente }\end{array}$ & $\begin{array}{c}\text { Concordo } \\
\text { parcialmente }\end{array}$ & Neutro & $\begin{array}{c}\text { Discordo } \\
\text { parcialmente }\end{array}$ & $\begin{array}{l}\text { Discordo } \\
\text { totalmente }\end{array}$ \\
\hline \multirow{2}{*}{ Controle } & $25 \%$ & $44 \%$ & \multirow{2}{*}{$19 \%$} & $9 \%$ & $3 \%$ \\
\hline & \multicolumn{2}{|c|}{$69 \%$} & & \multicolumn{2}{|c|}{$12 \%$} \\
\hline \multirow{2}{*}{ Experimental } & $39 \%$ & $36 \%$ & \multirow{2}{*}{$16 \%$} & $6 \%$ & $3 \%$ \\
\hline & & & & \multicolumn{2}{|c|}{$9 \%$} \\
\hline
\end{tabular}

Questão 3 - Você se sentiu confortável em avaliar os colegas que lhe foram atribuídos para a avaliação?

\begin{tabular}{c|c|c|c|c|c}
\hline \multirow{2}{*}{ Controle } & $\begin{array}{c}\text { Concordo } \\
\text { totalmente }\end{array}$ & $\begin{array}{c}\text { Concordo } \\
\text { parcialmente }\end{array}$ & Neutro & $\begin{array}{c}\text { Discordo } \\
\text { parcialmente }\end{array}$ & $\begin{array}{c}\text { Discordo } \\
\text { totalmente }\end{array}$ \\
\cline { 2 - 3 } & $41 \%$ & $31 \%$ & \multirow{2}{*}{$22 \%$} & $6 \%$ & $0 \%$ \\
\cline { 2 - 3 } Experimental & $52 \%$ & $26 \%$ & \multirow{2}{*}{$16 \%$} & \multicolumn{2}{|c}{$6 \%$} \\
\cline { 2 - 3 } \cline { 5 - 6 } & \multicolumn{2}{|c|}{$81 \%$} & & \multicolumn{2}{|c}{$3 \%$} \\
\hline
\end{tabular}

Questão 4 - Você se sentiu confortável ao ser avaliado(a) pelos colegas que corrigiram suas atividades?

\begin{tabular}{c|c|c|c|c|c}
\hline \multirow{2}{*}{ Controle } & $\begin{array}{c}\text { Concordo } \\
\text { totalmente }\end{array}$ & $\begin{array}{c}\text { Concordo } \\
\text { parcialmente }\end{array}$ & Neutro & $\begin{array}{c}\text { Discordo } \\
\text { parcialmente }\end{array}$ & $\begin{array}{c}\text { Discordo } \\
\text { totalmente }\end{array}$ \\
\cline { 2 - 3 } & $35 \%$ & $34 \%$ & \multirow{2}{*}{$19 \%$} & $6 \%$ & $6 \%$ \\
\cline { 2 - 3 } Experimental & $29 \%$ & $39 \%$ & \multirow{2}{*}{$26 \%$} & \multicolumn{2}{|c}{$12 \%$} \\
\cline { 2 - 3 } \cline { 5 - 6 } & \multicolumn{2}{|c|}{$68 \%$} & & \multicolumn{2}{|c}{$6 \%$} \\
\hline
\end{tabular}

Com relação à segunda pergunta de pesquisa, a Tabela 2 apresenta as questões e porcentagens obtidas. Constatou-se, com base na questão 5 , que os estudantes do grupo experimental discordaram em maior quantidade quanto a relevância dos apontamentos recebidos pelos pares avaliadores (36\%) em relação ao grupo de controle (19\%). Os estudantes do grupo controle também concordaram em maior número quanto a relevância dos apontamentos (65\%) com relação ao grupo experimental (48\%).

A questão 6, quanto a percepção de diferentes perspectivas das avaliações recebidas, demonstrou que $79 \%$ dos estudantes do grupo de controle concordou que houve diferentes perspectivas, enquanto no grupo experimental $75 \%$ concordaram. Apesar de uma maioria no grupo de controle concordar com a questão em relação ao grupo experimental, no grupo de controle há uma maior porcentagem de estudantes que discordam da questão (12\%) em relação ao grupo experimental (6\%).

Buscou-se compreender os resultados obtidos sobre a percepção discente quanto à segunda pergunta de pesquisa. Relacionando a questão 5 , verificou-se que em apenas $4,8 \%$ dos casos os estudantes do grupo experimental receberam um feedback escrito da avaliação e $6,4 \%$ no grupo de controle. Havia ainda a possibilidade de realizar comentários em cada uma das rubricas e em apenas $2,7 \%$ dos casos os comentários foram feitos no grupo experimental e $3,2 \%$ no grupo de controle. 
VIII Congresso Brasileiro de Informática na Educação (CBIE 2019)

Anais do XXX Simpósio Brasileiro de Informática na Educação (SBIE 2019)

Os estudantes, no geral, se limitaram apenas a selecionar o peso das rubricas propostas, que era obrigatório no sistema, sendo que ao questionarem a necessidade do feedback escrito foram informados que não era obrigatório mas que era relevante para a avalição de seus pares. Há uma dificuldade dos estudantes em relação a dar feedback qualitativo, diferente de avaliar com marcas, por exemplo [Sitthiworachart e Joy, 2008].

Estudos discutem que há grande importância na presença de justificativas junto as revisões dos pares para melhorar o desempenho da aprendizagem [Chew et al., 2016]. O experimento de Xiao e Lucking [2008] discute uma melhora nas habilidades dos estudantes cujo grupo recebia feedback qualitativo além de dados numéricos como avaliação assim como uma satisfação maior dos estudantes quanto ao OPA no geral.

Tabela 2. Resultados da Pergunta de Pesquisa 2

\begin{tabular}{|c|c|c|c|c|c|}
\hline & $\begin{array}{l}\text { Concordo } \\
\text { totalmente } \\
\end{array}$ & $\begin{array}{c}\text { Concordo } \\
\text { parcialmente }\end{array}$ & Neutro & $\begin{array}{c}\text { Discordo } \\
\text { parcialmente }\end{array}$ & $\begin{array}{l}\text { Discordo } \\
\text { totalmente } \\
\end{array}$ \\
\hline \multirow{2}{*}{ Controle } & $28 \%$ & $37 \%$ & \multirow{2}{*}{$16 \%$} & $19 \%$ & $0 \%$ \\
\hline & \multicolumn{2}{|c|}{$65 \%$} & & \multicolumn{2}{|c|}{$19 \%$} \\
\hline \multirow{2}{*}{ Experimental } & $19 \%$ & $29 \%$ & \multirow{2}{*}{$16 \%$} & $29 \%$ & $7 \%$ \\
\hline & & & & \multicolumn{2}{|c|}{$36 \%$} \\
\hline \multicolumn{6}{|c|}{$\begin{array}{l}\text { Questão } 6 \text { - Você percebeu diferentes perspectivas dos seus pares avaliadores para melhorar o } \\
\text { seu trabalho? Ou seja, cada colega trouxe diferentes contribuições para seu trabalho. }\end{array}$} \\
\hline & $\begin{array}{l}\text { Concordo } \\
\text { totalmente }\end{array}$ & $\begin{array}{c}\text { Concordo } \\
\text { parcialmente }\end{array}$ & Neutro & $\begin{array}{c}\text { Discordo } \\
\text { parcialmente }\end{array}$ & $\begin{array}{c}\text { Discordo } \\
\text { totalmente }\end{array}$ \\
\hline \multirow{2}{*}{ Controle } & $41 \%$ & $38 \%$ & \multirow{2}{*}{$9 \%$} & $6 \%$ & $6 \%$ \\
\hline & \multicolumn{2}{|c|}{$79 \%$} & & \multicolumn{2}{|c|}{$12 \%$} \\
\hline \multirow{2}{*}{ Experimental } & $39 \%$ & $36 \%$ & \multirow{2}{*}{$19 \%$} & $6 \%$ & $0 \%$ \\
\hline & & & & \multicolumn{2}{|c|}{$6 \%$} \\
\hline
\end{tabular}

Além dos dados debatidos acima, a questão 8 (Participar de avaliações pelos pares contribuiu para minha aprendizagem, porque...) trouxe informações relevantes quanto ao benefício aos estudantes no emprego do processo da OPA. Parte dos estudantes dos grupos de controle e experimental citam que a contribuição da OPA ocorreu no momento em que realizavam a avaliação de seus pares. Relataram que, ao compararem seu próprio trabalho com o de seus colegas, percebem tanto informações adicionais necessárias quanto falhas que não deveriam repetir em suas atividades.

Algumas transcrições referentes as afirmações dos estudantes: "Podemos reavaliar nossa atividade e aprender ao corrigir a de terceiros"; "Sim, pois pude observar outros trabalhos, que refisseram (sic) minhas ideias em relação ao meu próprio trabalho"; "Eu pude ver como os colegas fizeram e vi onde acertei, errei e onde fui regular"; "Porque ao avaliar outras provas vi diferentes pontos de vista para um mesmo problema"; "Sim, porque ao avaliar outros exercícios, acabo vendo os meus erros e como posso fazer para melhorar". Os relatos dos estudantes vão de encontro as percepções prévias dos pesquisadores deste estudo quanto ao processo da OPA, pois acreditava-se na contribuição educacional da OPA relacionada ao feedback final.

Destaca-se que, na questão 7 , foi perguntado aos estudantes se sentiram dificuldades durante o processo da OPA. No grupo de controle cerca de $20 \%$ dos 
VIII Congresso Brasileiro de Informática na Educação (CBIE 2019)

Anais do XXX Simpósio Brasileiro de Informática na Educação (SBIE 2019)

estudantes relataram alguma dificuldade (dentre eles, um estudante cita a dificuldade em avaliar seus colegas), frente a $10 \%$ do grupo experimental.

\section{Considerações Finais}

É importante destacar que este experimento levanta a necessidade de novos estudos. Foram envolvidos inicialmente 63 estudantes para trabalhar as perguntas de pesquisa quanto à heterogeneidade dos pares a partir das variáveis escolhidas (notas e perfil de personalidade). Entre os trabalhos futuros, é necessário investigar outras variáveis, como a análise de outras características e atributos na formação dos pares, objetivando coletar mais dados que aprofundem a discussão e fundamentem a pesquisa com maior precisão.

Estudos tem empregado tecnologia computacional no suporte ao processo da avaliação por pares, como é o caso de [Alves et al., 2018], demonstrando a importância dos estudos na área. A diversidade trazida pela formação heterogênea ajuda o estudante a ter acesso a avaliações, assim como ao avaliar as atividades, de outros estudantes com variados pontos de vista e organização de ideias para a resolução dos problemas propostos. Podem assim aplicar as novas perspectivas em suas próprias atividades. Os resultados iniciais vão ao encontro das necessidades de compreender, abordar e trabalhar com questões de desconfortos relacionadas a OPA, conforme citam [Kaufman e Schunn, 2011], contribuindo para estudos subsequentes da OPA.

\section{Agradecimentos}

O presente trabalho foi realizado com apoio da Coordenação de Aperfeiçoamento de Pessoal de Nível Superior - Brasil (CAPES) - Código de Financiamento 001.

\section{Referências}

Acharya, A e Sinha, D. A (2018) "Mixed" Strategy for Collaborative Group Formation and Its Learning Outcomes. Journal of Educational Technology Systems, v. 46, n. 4, p. $440-462$.

Alves, J., Silva, W., Brito, J. O., e Oliveira, E. (2018) Avaliação em Pares e Autoavaliação: Um Modelo Estatístico Para Perfilação de Alunos. In: Brazilian Symposium on Computers in Education (Simpósio Brasileiro de Informática na Educação-SBIE), p. 1653-1662.

Balmaceda, J. M., Schiaffino, S. N. e Pace, J. A. D. (2014) Using constraint satisfaction to aid group formation in CSCL. Inteligencia Artificial, Revista Iberoamericana de Inteligencia Artificial, v. 17, n. 53, p. 35-45.

Bouzidi, L. e Jaillet, A. (2009) Can online peer assessment be trusted?. Journal of Educational Technology \& Society, v. 12, n. 4, p. 257.

Cassidy, S. (2007) Assessing 'inexperienced'students' ability to self-assess: Exploring links with learning style and academic personal control. Assessment \& Evaluation in Higher Education, v. 32, n. 3, p. 313-330.

Chen, C. (2010) The implementation and evaluation of a mobile self-and peerassessment system. Computers \& Education, v. 55, n. 1, p. 229-236. 
VIII Congresso Brasileiro de Informática na Educação (CBIE 2019)

Anais do XXX Simpósio Brasileiro de Informática na Educação (SBIE 2019)

Chew, E, Snee, H. e Price, T. (2016) Enhancing international postgraduates' learning experience with online peer assessment and feedback innovation. Innovations in Education and Teaching International, v. 53, n. 3, p. 247-259.

Coutinho, C. (2011) Metodologias de investigação em ciências humanas. Coimbra: Almedina.

Couto, G., Bartholomeu, D. e Montiel, J. M. (2016) Estrutura interna do Myers Briggs Type Indicator (MBTI): evidência de validade. Avaliação Psicológica, v. 15, n. 1, p. 41-48.

Demir, M. (2018) Using online peer assessment in an Instructional Technology and Material Design course through social media. Higher Education, v. 75, n. 3, p. 399414.

Dillenbourg, P. (1999) What do you mean by collaborative learning? Elsevier.

Fernandes, D. (2005) Avaliação alternativa: perspectivas teóricas e práticas de apoio. Livro do $3 .{ }^{\circ}$ Congresso Internacional Sobre Avaliação na Educação. Futuro Eventos, p. 79-92.

Garcia, J. (2009) Avaliação e aprendizagem na educação superior. Estudos em Avaliação Educacional, v. 20, n. 43, p. 201-213.

Gokhale, A. A. (2012) Collaborative learning and critical thinking. Encyclopedia of the sciences of learning, p. 634-636.

Ho, T. F., Shyu, S. J., Wang, F. H., e Li, C. T. J. (2009) Composing high-heterogeneous and high-interaction groups in collaborative learning with particle swarm optimization. Computer Science and Information Engineering, 2009 WRI World Congress. IEEE, p. 607-611.

Kardan, A. A. e Sadeghi, H. (2016) An efficacious dynamic mathematical modelling approach for creation of best collaborative groups. Mathematical and Computer Modelling of Dynamical Systems, v. 22, n. 1, p. 39-53.

Kaufman, J. H. e Schunn, C. D. (2011) Students' perceptions about peer assessment for writing: their origin and impact on revision work. Instructional Science, v. 39, n. 3, p. 387-406.

Khandaker, N. e Soh, L. (2010) Improving group selection and assessment in an asynchronous collaborative writing application. International Journal of Artificial Intelligence in Education, v. 20, n. 3, p. 231-268.

Lai, C. (2016) Training nursing students' communication skills with online video peer assessment. Computers \& Education, v. 97, p. 21-30.

Li, L., Liu, X. e Zhou, Y. (2012). Give and take: A re-analysis of assessor and assessee's roles in technology-facilitated peer assessment. British Journal of Educational Technology, 43(3), 376-384.

Ma, A. W. W. (2009) A Longitudinal Study of the Use of Computer Supported Collaborative Learning in Promoting Lifelong Learning Skills. Issues in Informing Science \& Information Technology, v. 6. 
VIII Congresso Brasileiro de Informática na Educação (CBIE 2019)

Anais do XXX Simpósio Brasileiro de Informática na Educação (SBIE 2019)

Maina, E. M., Oboko, R. O. e Waiganjo, P. W. (2017) Using Machine Learning Techniques to Support Group Formation in an Online Collaborative Learning Environment. International Journal of Intelligent Systems \& Applications, v. 9, n. 3.

Miao, Y. e Koper, R. (2007) An efficient and flexible technical approach to develop and deliver online peer assessment. 8th international conference on Computer supported collaborative learning. International Society of the Learning Sciences, p. 506-515.

Pereira, A., Oliveira, I. e Tinoca, L. (2010) A cultura de avaliação: que dimensões?. I Encontro Internacional TIC e Educação.

Reis, R. C. D., Isotani, S., Rodriguez, C. L., Lyra, K. T., Jaques, P. A. e Bittencourt, I. I. (2018). Affective states in computer-supported collaborative learning: Studying the past to drive the future. Computers \& Education, 120, 29-50.

Sáiz, M. S. I., Gomez, G. R. e Ruiz, M. A. G. (2012) Benefits of peer assessment and strategies for its practice at university. Revista de Educación, n. 359, p. 206-231.

Sitthiworachart, J. e Joy, M. (2008) Computer support of effective peer assessment in an undergraduate programming class. Journal of computer assisted learning, v. 24, n. 3, p. $217-231$.

Smith, J. V. e Spindle, R. M. (2007) The impact of group formation in a cooperative learning environment. Journal of Accounting Education, v. 25, n. 4, p. 153-167.

Stahl, G., Koschmann, T. D. e Suthers, D. D. (2006) Computer-supported collaborative learning, p. 409-426.

To, J. (2016) 'This is not what I need': conflicting assessment feedback beliefs in a post-secondary institution in Hong Kong. Research in Post-Compulsory Education, v. 21, n. 4, p. 447-467.

Topping, K. J. (2009) Peer assessment. Theory into practice, v. 48, n. 1, p. 20-27.

Van der Pol, J., Van den Berg, B. A. M., Admiraal, W. F., e Simons, P. R. J. (2008) The nature, reception, and use of online peer feedback in higher education. Computers \& Education, v. 51, n. 4, p. 1804-1817.

Wagstaff, K., Cardie, C., Rogers, S., e Schrödl, S. (2001) Constrained k-means clustering with background knowledge. ICML, p. 577-584.

Webber, C. G. e Lima, M. F. W. P. (2012) Evaluating automatic group formation mechanisms to promote collaborative learning-a case study. International Journal of Learning Technology, v. 7, n. 3, p. 261-276.

Wen, M. L. e Tsai, C. (2008) Online peer assessment in an inservice science and mathematics teacher education course. Teaching in Higher Education, v. 13, n. 1, p. 55-67.

Xiao, Y. e Lucking, R. (2008) The impact of two types of peer assessment on students' performance and satisfaction within a Wiki environment. The Internet and Higher Education, v. 11, n. 3-4, p. 186-193.

Yang, Y. e Tsai, C. (2010) Conceptions of and approaches to learning through online peer assessment. Learning and Instruction, v. 20, n. 1, p. 72-83. 